Supplementary Information

\title{
Comparison of cytotoxicity evaluation of anticancer drugs between real-time cell analysis and CCK-8 method
}

\author{
Ling Cai ${ }^{1 \#}$, Xijiang Qin ${ }^{1 \#}$, Zhihui Xu1 , Yiyan Song ${ }^{1}$, Huijun Jiang ${ }^{2}$, \\ Yuan $\mathrm{Wu}^{3}$, Hongjie Ruan ${ }^{4}$, Jin Chen ${ }^{1,5,6^{*}}$
}

${ }^{1}$ School of Public Health, Nanjing Medical University, Nanjing 211166, China

${ }^{2}$ School of Pharmacy, Nanjing Medical University, Nanjing 211166, China

${ }^{3}$ Department of Medical Oncology, Jiangsu Cancer Hospital, Jiangsu Institute of Cancer Research, The Affiliated Cancer Hospital of Nanjing Medical University, Nanjing 210009, China

${ }^{4}$ Department of gynecology, Obstetrics and Gynecology Hospital Affiliated to Nanjing Medical University, Nanjing, 210004, China

${ }^{5}$ The Key Laboratory of Modern Toxicology, Ministry of Education, School of Public Health, Nanjing Medical University, Nanjing 211166, Jiangsu, China ${ }^{6}$ Center for Global Health, School of Public Health, Nanjing Medical University, Nanjing 211166, Jiangsu, China

${ }^{*}$ Correspondence and request for materials should be addressed to J.C. (email: okachen30@gmail.com; jchen@njmu.edu.cn)

\#Equal contribution 
A
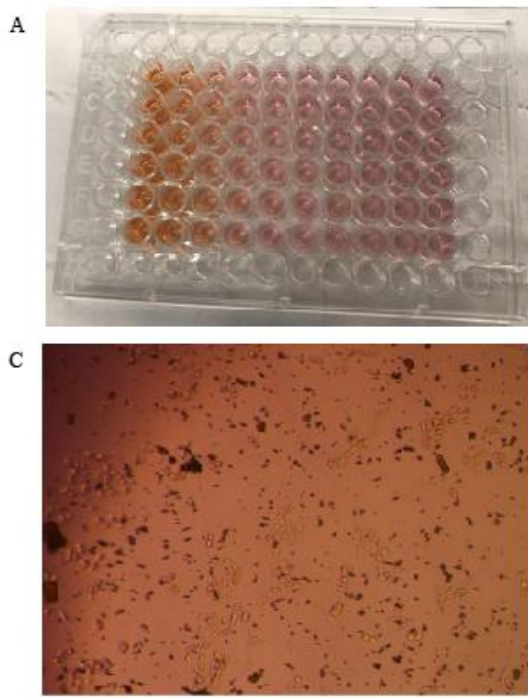
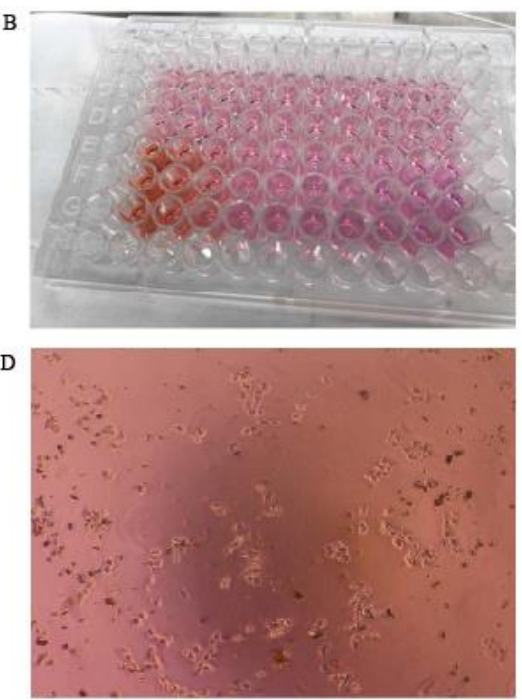

Figure S1 Images of HeLa cultured with curcumin. Photograghs of the 96-well plate containing (A) HeLa cells after 24h-effection of a series concentrations of curcumin and (B) curcumin-effected HeLa cells, of which row B, C and D were rinsed with fresh DMEM while row E, F and $\mathrm{G}$ remained unchanged. Images of the cell sample from the same 96-well plate containing $200 \mu \mathrm{g} / \mathrm{mL}$ curcumin before (C) and after (D) replacing the culture medium.
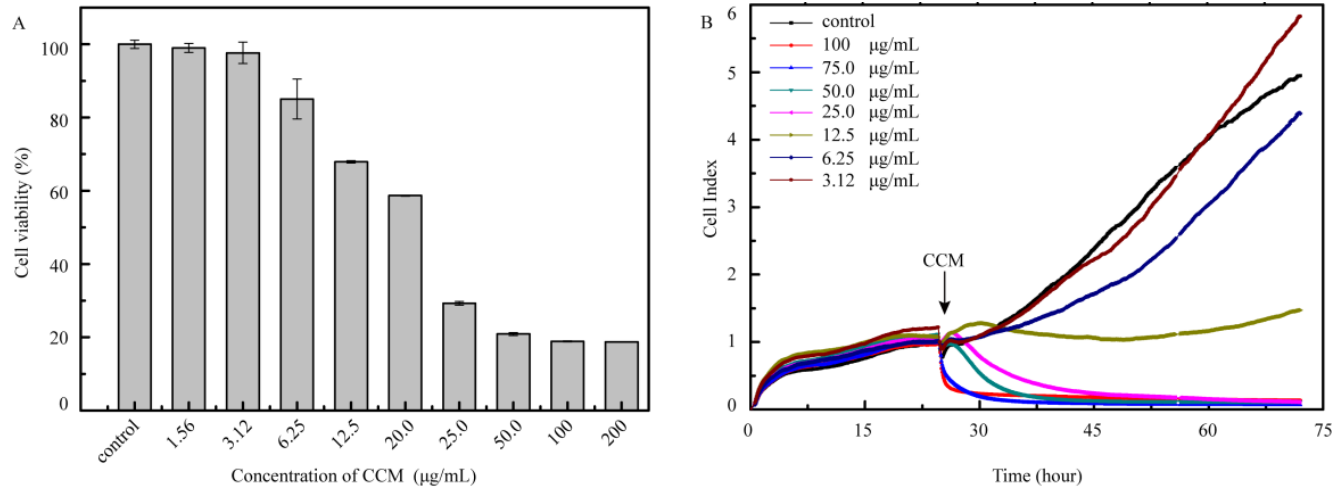

Figure S2 (A) Cell viability of A549 induced by CCM after $24 \mathrm{~h}$ in CCK-8 assay; (B) cell growth curve of A549 induced by CCM in RTCA. 

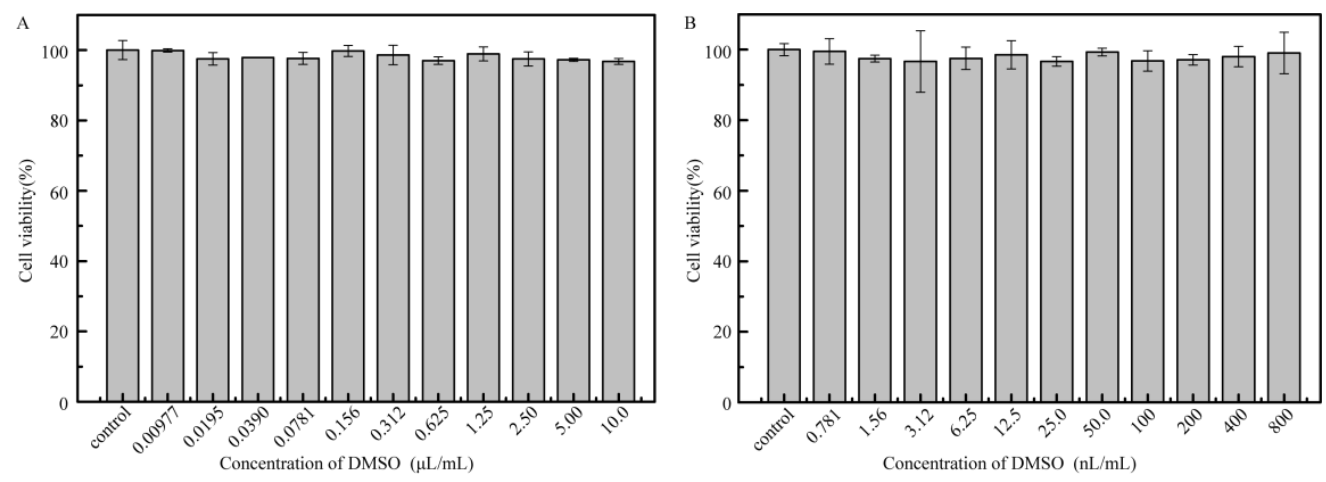

Figure S3 Cell viability of HeLa cells induced by DMSO after $24 \mathrm{~h}$ (A) and $48 \mathrm{~h}$ (B) in CCK-8 assay. 